Article

\title{
Qualitative and Quantitative Analysis of the Major Constituents in WLJ Herbal Tea Using Multiple Chromatographic Techniques
}

\author{
Chao-Zhan Lin ${ }^{1}{ }^{(}$, Run-Jing Zhang ${ }^{1}$, Yu-Feng Yao ${ }^{1}$, Xiao-Dan Huang ${ }^{2, *}$, Rong-Bo Zheng ${ }^{2}$, \\ Bo-Jian $\mathrm{Wu}^{2}$ and Chen-Chen $\mathrm{Zhu}{ }^{1, *}$ \\ 1 Institute of Clinical Pharmacology, Guangzhou University of Chinese Medicine, No. 12 Jichang Road, \\ Guangzhou 510405, China; linchaozhan@gzucm.edu.cn (C.-Z.L.); zhang_runjing@sina.com (R.-J.Z.); \\ yao_yufeng@sina.com (Y.-F.Y.) \\ 2 Research \& Development Institute, Guangzhou Wanglaoji Pharmaceutical Company Limited, No. 831 \\ Guanghuaer Road, Guangzhou 510450, China; zrbb0819@sina.com (R.-B.Z.); wu_bojian@sina.com (B.-J.W.) \\ * Correspondence: xiaodanhuang@126.com (X.-D.H.); zhucc@gzucm.edu.cn (C.-C.Z.); \\ Tel.: +86-2066830466 (X.-D.H.); +86-2036585303 (C.-C.Z.); \\ Fax: +86-2086601051 (X.-D.H.); +86-2036588015 (C.-C.Z.)
}

Received: 29 August 2018; Accepted: 11 October 2018; Published: 12 October 2018

\begin{abstract}
Quality control of Chinese herbal tea remains a challenge due to our poor knowledge of their complex chemical profile. This study aims to investigate the chemical composition of one of the best-selling and famous brand of beverage in China, Wanglaoji Herbal Tea (WLJHT), via a full component quantitative analysis. In this paper, a total of thirty-four representative constituents were identified or tentatively characterized using ultra-high performance liquid chromatography coupled with quadrupole tandem time-of-flight mass spectrometry (UPLC-Q-TOF-MS). Moreover, the quantitative analyses of fourteen constituents were performed by high performance liquid chromatography with a triple quadruple tandem mass spectrometry (HPLC-MS/MS) method and saccharide compositions of WLJHT were also quantitatively determined by high performance liquid chromatography (HPLC) with evaporative light scattering detector (ELSD) on a Hilic column, separately. Using multiple chromatographic techniques presented a good precision, sensitivity, repeatability and stability, and was successfully applied to analyze 16 batches of WLJHT samples. Therefore, it would be a reliable and useful approach for the quality control of WLJHT.
\end{abstract}

Keywords: Wanglaoji herbal tea; UPLC-Q-TOF-MS; HPLC-MS/MS; evaporative light scattering detector

\section{Introduction}

Traditional Chinese herbal tea, developed by Chinese people during the long-term for disease prevention and health care under the guidance of traditional Chinese medicine, has been approved as an intangible cultural heritage by the Chinese government in 2006 [1-3].

Wanglaoji Herbal Tea (WLJHT), founded in the Daoguang eighth year of Qing Dynasty (Ad 1828), is the earliest Cantonese herbal tea and recognized as the ancestor of herbal tea. It is consisted of seven traditional Chinese medicines (Chinese Mesona, PlumeriaeFlos, LoniceraeJaponicaeFlos, ChrysanthemiFlos, Prunellae Spica, Microctis Folium and Glycyrrhizae Radix et Rhizoma), and widely used for heat-clearing, detoxicating, engender liquid and allay thirst. Modern pharmacological studies have proven its protective effects on liver damage [2], improvement of cytotoxic T Lymphocytes activity in spleen [4], amelioration on lipids metabolism [5] and glucometabolism [6], and enhancement of immune functions of restrain-stress mice [7]. In our previous phytochemical study [8], several polyphenol constituents as phenolic acids and flavonoids were separated and structural elucidated from WLJHT. 
As "King of herbal tea", WLJHT is lack of effective approach for quality control due to its chemical complexity. Three phenolic acids have been suggested to be employed for the quality assessment of WLJHT. However, as is known to all, multi-components in compositive herbs attribute comprehensive efficacy of herbal tea. Therefore, quality assessment based on a few of markers has been proven to be insufficient. In consideration of a mounts of unknown chemicals existed in herbal tea, LC/MS was widely used for the quality analyses of herbal medicines and herbal teas due to its powerful function on chemical structures [9]. Meanwhile, with the limitation of MS on carbohydrates, the evaporative light scattering detector (ELSD) has been used as an efficient method to identify and determine the little molecular carbohydrates in multi-herbals [10-14]. Therefore, complex application of multiple techniques for quality assessment of herbal tea seems to be more feasible and effective.

The aim of this study is to identify and quantify of the major constituents both the small molecules and saccharides in WLJHT, using ultra-high performance liquid chromatography (UPLC) coupled with quadrupole tandem time-of-flight mass spectrometry (Q-TOF-MS), and high performance liquid chromatography equipped with evaporative light scattering detector (HPLC-ELSD). 16 batches of samples were analyzed and the results were expected to provide comprehensive information for the quality control of WLJHT.

\section{Results and Discussion}

\subsection{Identification of Constituents in WLJHT}

Both positive and negative ion modes were detected for MS analysis depending on the different chemical properties of WLJHT as shown in Figure 1A,B, respectively. In the negative-ion ESI mode experiments, the deprotonated molecules $[\mathrm{M}-\mathrm{H}]^{-}$were detected as the base peaks for most of the constituents.
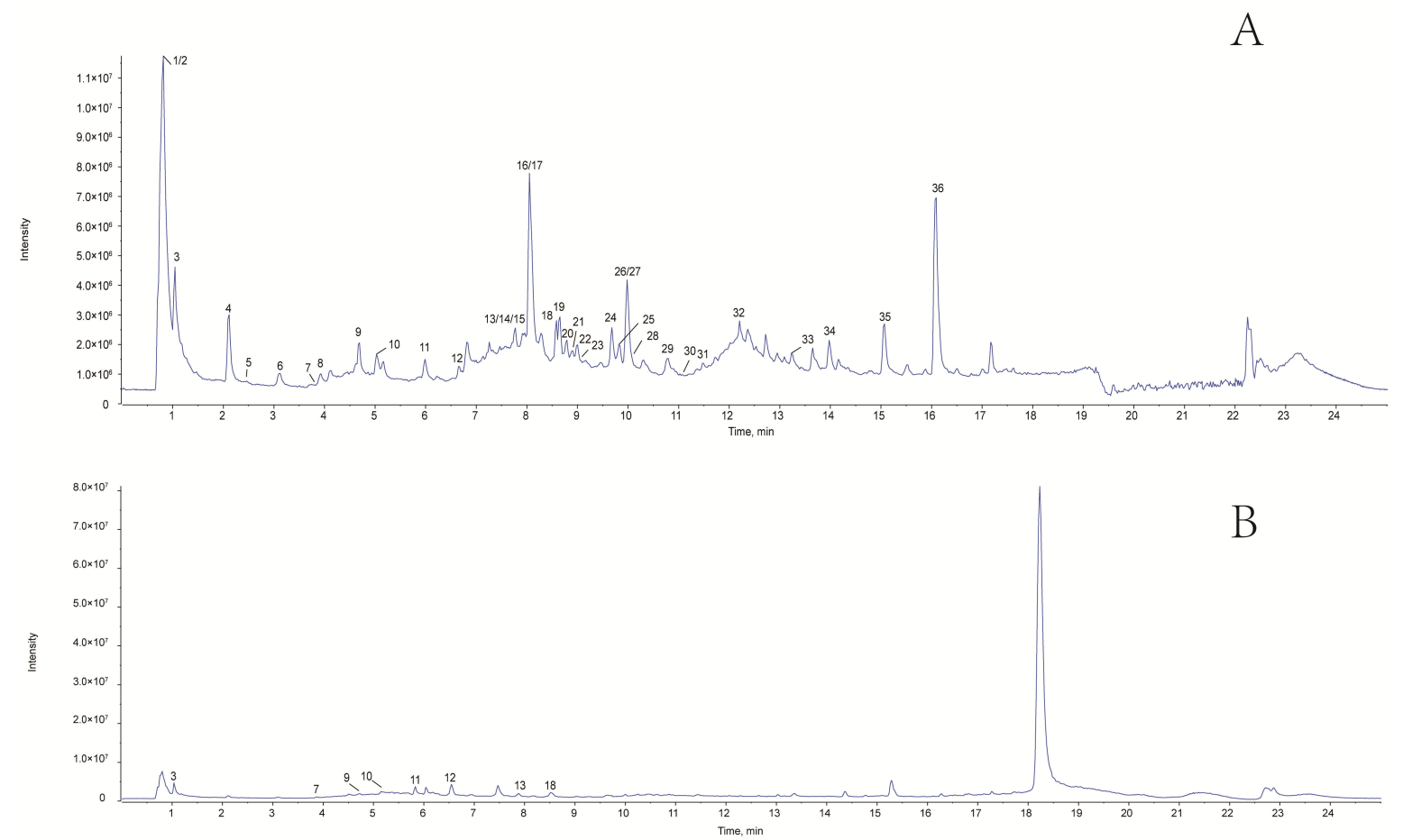

Figure 1. Representative total ion chromatograms of the WLJHT. (A) TIC of WLJHT sample in negative ion mode. (B) TIC of WLJHT sample in positive ion mode. 
Table 1. Identification of chemical constituents of WLJ herbal tea by UPLC/Q-TOF-MS/MS in positive and negative ion modes.

\begin{tabular}{|c|c|c|c|c|c|}
\hline Peak & $t_{R}(\min )$ & $\begin{array}{l}\text { Molecular } \\
\text { Formula }\end{array}$ & $\begin{array}{l}\text { Experimental Weight of Positive ESI-TOF-MS } \\
(\mathrm{m} / \mathrm{z}) / \text { Error }(\mathrm{ppm}) / \text { fragmental ion }\end{array}$ & $\begin{array}{l}\text { Experimental Weight of Negative ESI-TOF-MS } \\
(\mathrm{m} / \mathrm{z}) / \text { Error }(\mathrm{ppm}) / \text { Fragmental Ion }\end{array}$ & Identification \\
\hline 1 & 0.80 & $\mathrm{C}_{12} \mathrm{H}_{22} \mathrm{O}_{11}$ & $343.1238[\mathrm{M}+\mathrm{H}]^{+} / 0.9$ & $341.1089[\mathrm{M}-\mathrm{H}]^{-} /-0.1$ & Disaccharide \\
\hline 2 & 0.83 & $\mathrm{C}_{6} \mathrm{H}_{12} \mathrm{O}_{7}$ & $343.1238[\mathrm{M}+\mathrm{H}]^{+} / 0.9 / 240,183$ & $341.1089[\mathrm{M}-\mathrm{H}]^{-} /-0.1 / 179,161,143,113$ & Glucose acid \\
\hline 3 & 1.04 & $\mathrm{C}_{6} \mathrm{H}_{8} \mathrm{O}_{7}$ & Null ${ }^{c}$ & $195.0514[\mathrm{M}-\mathrm{H}]^{-} / 2.2 / 96,87,75$ & Citricacid ${ }^{b},[15]$ \\
\hline 4 & 2.11 & $\mathrm{C}_{7} \mathrm{H}_{6} \mathrm{O}_{4}$ & Null & $191.0202[\mathrm{M}-\mathrm{H}]^{-} / 0.8 / 111,87,85,67$ & Protocatechuid acid ${ }^{\mathrm{a}}$ \\
\hline 5 & 2.45 & $\mathrm{C}_{7} \mathrm{H}_{6} \mathrm{O}_{3}$ & $155.0338[\mathrm{M}+\mathrm{H}]^{+} /-0.5 / 85,83,56$ & $153.0198\left[\mathrm{M}-\mathrm{H}^{-} / 3.0 / 109,91,81\right.$ & Protocatechualdehyde ${ }^{a}$ \\
\hline 6 & 3.08 & $\mathrm{C}_{16} \mathrm{H}_{18} \mathrm{O}_{9}$ & $139.0399[\mathrm{M}+\mathrm{H}]^{+} / 7.0 / 95$ & $137.0249[\mathrm{M}-\mathrm{H}]^{-} / 3.8 / 93,65$ & 5-O-Caffeoylquinic acid ${ }^{b},[9]$ \\
\hline 7 & 3.92 & $\mathrm{C}_{20} \mathrm{H}_{24} \mathrm{O}_{12}$ & Null & $353.0872[\mathrm{M}-\mathrm{H}]^{-} / 0.9 / 191,179,135$ & 15-Demethylplumieride ${ }^{\text {a }}$ \\
\hline 8 & 3.95 & $\mathrm{C}_{9} \mathrm{H}_{10} \mathrm{O}_{5}$ & $457.1333[\mathrm{M}+\mathrm{H}]^{+} /-1.5 / 277$ & $455.1188[\mathrm{M}-\mathrm{H}]^{-} /-1.4 / 275,231$ & Syringic acid ${ }^{\mathrm{a}}$ \\
\hline 9 & 4.68 & $\mathrm{C}_{16} \mathrm{H}_{18} \mathrm{O}_{9}$ & $199.0597[\mathrm{M}+\mathrm{H}]^{+} /-1.8 / 139,135,107$ & $197.0459[\mathrm{M}-\mathrm{H}]^{-} / 2.2 / 135,123$ & Chlorogenic acid ${ }^{\text {a }}$ \\
\hline 10 & 5.12 & $\mathrm{C}_{9} \mathrm{H}_{8} \mathrm{O}_{4}$ & $355.1026[\mathrm{M}+\mathrm{H}]^{+} / 0.8 / 163,145$ & $353.0874[\mathrm{M}-\mathrm{H}]^{-} /-0.8 / 191,85$ & Caffeic acid ${ }^{a}$ \\
\hline 11 & 5.99 & $\mathrm{C}_{27} \mathrm{H}_{30} \mathrm{O}_{16}$ & $181.0492[\mathrm{M}+\mathrm{H}]^{+} /-1.4 / 145,135,89$ & $179.0351[\mathrm{M}-\mathrm{H}]^{-} / 0.7 / 131,85,71$ & Rutin $^{\mathrm{a}}$ \\
\hline 12 & 6.80 & $\mathrm{C}_{25} \mathrm{H}_{24} \mathrm{O}_{12}$ & $611.1597[\mathrm{M}+\mathrm{H}]^{+} /-1.5 / 566,548$ & $609.1454[\mathrm{M}-\mathrm{H}]^{-} /-1.1 / 301$ & Isomeric di-O-CQA \\
\hline 13 & 7.91 & $\mathrm{C}_{10} \mathrm{H}_{10} \mathrm{O}_{5}$ & $517.1324[\mathrm{M}+\mathrm{H}]^{+} /-3.0 / 499,319,163$ & $515.1188[\mathrm{M}-\mathrm{H}]^{-} /-1.2 / 353,191,179$ & Cerberic acid $\mathrm{B}^{\mathrm{a}}$ \\
\hline 14 & 7.91 & $\mathrm{C}_{27} \mathrm{H}_{30} \mathrm{O}_{14}$ & $211.0597[\mathrm{M}+\mathrm{H}]^{+} /-1.5 / 193$ & $209.0458[\mathrm{M}-\mathrm{H}]^{-} / 1.4 / 165,121,119$ & Violanthin ${ }^{\mathrm{b}}$, [16] \\
\hline 15 & 7.92 & $\mathrm{C}_{27} \mathrm{H}_{30} \mathrm{O}_{14}$ & $579.1708[\mathrm{M}+\mathrm{H}]^{+} /-1.1 / 379,337,325$ & $577.1556[\mathrm{M}-\mathrm{H}]^{-} /-0.3 / 457,353$ & Isoviolanthin \\
\hline 16 & 8.05 & $\mathrm{C}_{21} \mathrm{H}_{22} \mathrm{O}_{9}$ & $579.1701[\mathrm{M}+\mathrm{H}]^{+} /-1.1 / 507,447,337$ & $577.1557[\mathrm{M}-\mathrm{H}]^{-} /-0.8 / 503,473,457,413$ & Liquiritin $^{\text {a }}$ \\
\hline 17 & 8.09 & $\mathrm{C}_{24} \mathrm{H}_{26} \mathrm{O}_{13}$ & $419.1336[\mathrm{M}+\mathrm{H}]^{+} /-0.1 / 257,137$ & $417.1183[\mathrm{M}-\mathrm{H}]^{-} /-1.7 / 255,148$ & Rosmarinic acid glycoside $^{a}$ \\
\hline 18 & 8.58 & $\mathrm{C}_{30} \mathrm{H}_{32} \mathrm{O}_{15}$ & $523.1437[\mathrm{M}+\mathrm{H}]^{+} /-1.6 / 181,163$ & $521.1294[\mathrm{M}-\mathrm{H}]^{-} /-1.0 / 359,323$ & Caffeoylplumieride $^{\mathrm{a}}$ \\
\hline 19 & 8.65 & $\mathrm{C}_{27} \mathrm{H}_{32} \mathrm{O}_{15}$ & $633.1777[\mathrm{M}+\mathrm{H}]^{+} /-5.7 / 546,474$ & $631.1647[\mathrm{M}-\mathrm{H}]^{-} /-3.3 / 601 / 439,163$ & $\begin{array}{l}\text { Keampferol-3-O- } \beta \text {-D-glucose-7-O- } \\
\alpha \text {-L-rhamnase }{ }^{\text {a }}\end{array}$ \\
\hline 20 & 8.78 & $\mathrm{C}_{28} \mathrm{H}_{32} \mathrm{O}_{16}$ & $597.1776[\mathrm{M}+\mathrm{H}]^{+} /-6.2 / 548,435$ & $595.1659[\mathrm{M}-\mathrm{H}]^{-} /-1.5 / 285$ & Narcissoside $^{a}$ \\
\hline 21 & 8.90 & $\mathrm{C}_{25} \mathrm{H}_{24} \mathrm{O}_{12}$ & $625.1754[\mathrm{M}+\mathrm{H}]^{+} /-1.3 / 317$ & $623.1612[\mathrm{M}-\mathrm{H}]^{-} /-0.7 / 315,300$ & Isochlorogenic acid C \\
\hline 22 & 8.99 & $\mathrm{C}_{21} \mathrm{H}_{20} \mathrm{O}_{11}$ & $517.1324[\mathrm{M}+\mathrm{H}]^{+} /-3.0 / 499,319,163$ & $515.1188[\mathrm{M}-\mathrm{H}]^{-} /-1.2 / 353,335,173$ & Trifolin $^{\mathrm{a}}$ \\
\hline 23 & 9.00 & $\mathrm{C}_{25} \mathrm{H}_{24} \mathrm{O}_{12}$ & $449.1077[\mathrm{M}+\mathrm{H}]^{+} /-0.2 / 287$ & $447.0923[\mathrm{M}-\mathrm{H}]^{-} /-2.1 / 284,255$ & Isochlorogenic acid $\mathrm{A}^{\mathrm{a}}$ \\
\hline 24 & 9.68 & $\mathrm{C}_{36} \mathrm{H}_{30} \mathrm{O}_{16}$ & $517.1324[\mathrm{M}+\mathrm{H}]^{+} /-3.0 / 499,319,163$ & $515.1188[\mathrm{M}-\mathrm{H}]^{-} /-1.2 / 353,191$ & Salvianolic acid B \\
\hline
\end{tabular}


Table 1. Cont.

\begin{tabular}{|c|c|c|c|c|c|}
\hline Peak & $t_{R}(\min )$ & $\begin{array}{l}\text { Molecular } \\
\text { Formula }\end{array}$ & $\begin{array}{l}\text { Experimental Weight of Positive ESI-TOF-MS } \\
(\mathrm{m} / \mathrm{z}) / \text { Error }(\mathrm{ppm}) / \text { fragmental ion }\end{array}$ & $\begin{array}{l}\text { Experimental Weight of Negative ESI-TOF-MS } \\
(\mathrm{m} / \mathrm{z}) / \text { Error }(\mathrm{ppm}) / \text { Fragmental Ion }\end{array}$ & Identification \\
\hline 25 & 9.83 & $\mathrm{C}_{48} \mathrm{H}_{68} \mathrm{O}_{5}$ & $719.1604[\mathrm{M}+\mathrm{H}]^{+} /-0.3 / 643,431$ & $717.1458[\mathrm{M}-\mathrm{H}]^{-} /-0.4 / 673,537,519$ & Not identify \\
\hline 26 & 9.98 & $\mathrm{C}_{25} \mathrm{H}_{24} \mathrm{O}_{12}$ & $725.5148[\mathrm{M}+\mathrm{H}]^{+} / 1.2 / 661,643$ & $723.5029[\mathrm{M}-\mathrm{H}]^{-} / 4.8 / 677$ & Isochlorogenic acid B \\
\hline 27 & 9.98 & $\mathrm{C}_{18} \mathrm{H}_{16} \mathrm{O}_{8}$ & $517.1324[\mathrm{M}+\mathrm{H}]^{+} /-3.0 / 499,319,163$ & $515.1188[\mathrm{M}-\mathrm{H}]^{-} /-1.2 / 353,255,203,173$ & Rosmarinic acid $^{\text {a }}$ \\
\hline 28 & 10.09 & $\mathrm{C}_{26} \mathrm{H}_{22} \mathrm{O}_{10}$ & $361.0919[\mathrm{M}+\mathrm{H}]^{+} / 0.4 / 181,163$ & $359.0770[\mathrm{M}-\mathrm{H}]^{-} /-0.4 / 197,179,161$ & Salvianolic acid A \\
\hline 29 & 10.78 & $\mathrm{C}_{44} \mathrm{H}_{86} \mathrm{O}_{14}$ & $495.1225[\mathrm{M}+\mathrm{H}]^{+} /-2.2 / 297$ & $493.1120[\mathrm{M}-\mathrm{H}]^{-} /-4.0 / 295$ & Not identify \\
\hline 30 & 11.19 & $\mathrm{C}_{65} \mathrm{H}_{106} \mathrm{O}_{32}$ & $839.6083[\mathrm{M}+\mathrm{H}]^{+} /-0.8 / 661,351$ & $837.5897[\mathrm{M}-\mathrm{H}]^{-} /-5.7 / 791$ & Macranthoidin B ${ }^{a}$ \\
\hline 31 & 11.49 & $\mathrm{C}_{36} \mathrm{H}_{30} \mathrm{O}_{16}$ & $1399.671[\mathrm{M}+\mathrm{H}]^{+} /-2.0 / 1021,897,751$ & Null & Salvianolic acid E \\
\hline 32 & 12.20 & $\mathrm{C}_{30} \mathrm{H}_{46} \mathrm{O}_{8}$ & $719.1604[\mathrm{M}+\mathrm{H}]^{+} /-0.3 / 521,323$ & $717.1458[\mathrm{M}-\mathrm{H}]^{-} /-0.4 / 519$ & Neriifolin ${ }^{b},[17]$ \\
\hline 33 & 13.24 & $\mathrm{C}_{48} \mathrm{H}_{72} \mathrm{O}_{21}$ & Null & $579.3164[\mathrm{M}+\mathrm{COOH}]^{-} /-0.3 / 533,515,399$ & Licorice saponin A3 \\
\hline 34 & 13.99 & $\mathrm{C}_{53} \mathrm{H}_{86} \mathrm{O}_{22}$ & $985.4631[\mathrm{M}+\mathrm{H}]^{+} /-0.7 / 809,647,615$ & $983.4493[\mathrm{M}-\mathrm{H}]^{-} /-0.1 / 821$ & Dipsacoside B ${ }^{\text {a }}$ \\
\hline 35 & 15.05 & $\mathrm{C}_{42} \mathrm{H}_{62} \mathrm{O}_{17}$ & $1075.566[\mathrm{M}+\mathrm{H}]^{+} /-1.7 / 967,863$ & Null & Licorice saponin G2 \\
\hline 36 & 16.07 & $\mathrm{C}_{42} \mathrm{H}_{62} \mathrm{O}_{16}$ & $839.4053[\mathrm{M}+\mathrm{H}]^{+} /-0.7 / 663,487,469,451$ & $837.3916[\mathrm{M}-\mathrm{H}]^{-} / 0.3 / 351,193$ & Glycyrrhizic acid $^{\text {a }}$ \\
\hline
\end{tabular}

${ }^{a}$ The identity were confirmed by comparing its $\mathrm{t}_{\mathrm{R}}$, ESI-TOF-MS data with those of the reference substances. ${ }^{\mathrm{b}}$ Represented that compounds were identified with literatures. ${ }^{\mathrm{c}}$ Not detected. 
The exact molecular weight of each constituent was easily calculated according to the experimental mass of the pseudo-molecular ions, and the molecular formulas of those were deduced from each exact molecular weight obtained by Q-TOF-MS. The fragmentation information of each constituent was also obtained by Q-TOF-MS/MS as shown in Supplementary Information, which was quite useful for the identification of each constituent. Table 1 lists the retention time $\left(t_{R}\right)$, molecular formulas, experimental molecular weights, ESI-TOF-MS ions of thirty-four major peaks in the chromatograms came from WLJ herbal tea samples.

Among the thirty-six major constituents, a total of thirty-four constituents from the WLJHT were identified or tentatively characterized. They included 6 organic acids (Protocatechuid acid (4), Protocatechualdehyde (5), Chlorogenic acid (9), Caffeic acid (10), Isochlorogenic acid A (23), Rosmarinic acid (27)), 5 flavonoids (Rutin (11), Liquiritin (16), Keampferol-3-O- $\beta$-D-glucose-7-O- $\alpha$-L-rhamnase (19), Narcissoside (20), Trifolin (22)), 2 triterpenoids (Macranthoidin B (30), Dipsacoside B (34)), and 1 iridoid (caffeoylplumieride (18)) was unambiguously identified by comparison of their $t_{R} S$, ESI-IT-MS data with those of their reference substances. The other 20 compounds were tentatively characterized as follows: disaccharide (1), glucose acid (2), citric acid (3), 5-O-Caffeoylquinic acid (6), 15-demethylplumieride (7), syringic acid (8), isomeric di-O-CQA (10), cerberic acid B (13), violanthin (14), isoviolanthin (15), rosmarinic acid glycoside (17), isochlorogenic acid C (21), salvianolic acid B (24), isochlorogenic acid B (26), salvianolic acid A (28), salvianolic acid E (31), neriifolin (32), licorice saponin A3 (33), licorice saponin G2 (35), and glycyrrhizic acid (36) by comparing their exact molecular weights, MS $^{n}$ spectra, UV absorptions and retention behaviors with those of reported compounds [8,9,15-19].

On the other hand, the saccharide profile was also shown in Figure 2, in which only four saccharides-fructose, a/ $\beta$-D-glucose and sucrose-were identified by comparison with reference standards.
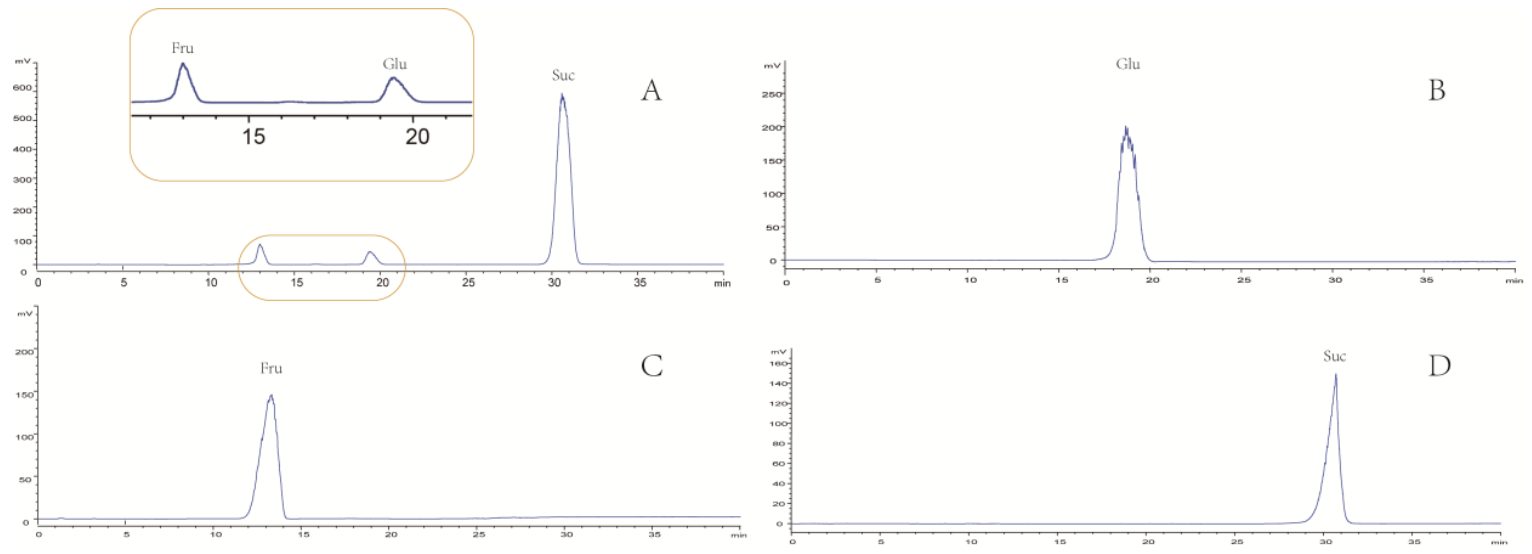

Figure 2. Comparative analysis of WLJHT (A) and 3 saccharides (B: Glucose; C: Fructose; D: Sucrose) using HPLC-ELSD couple with a Hilic column.

\subsection{Limitation of Qualitative Analysis Solely Using Mass Spectrometry}

WLJ herbal tea has two type components as non-sugar small molecules and little molecular carbohydrates. Although mass spectrometry is a powerful and sensitive analytical method as it can provide an accurate mass of the molecules and nanogram level of detection limit, solely using MS data in qualitative analysis is not suitable for weak electrolytes. For example, fructose, glucose and sucrose as the major carbohydrates of WLJHT, but they are too hard ionized to be detected by mass spectrometry. Therefore, it is suggested that the qualitative analysis is risky if only mass spectrometry is used. Moreover, the ionization mode plays an important role for MS analysis. The complex chemical composition of Chinese medicine requires a variety of ionization modes. Some components may be ignored if the single ionization mode is used. The comparison between two modes is necessary, which can avoid missing information. 


\subsection{Method Validation}

The linearity, ranges, regressions, LODs, LOQs and recoveries of the method are listed in Table 2. The data exhibited a satisfactory relationship between concentrations and peak areas of the analytes within the test ranges $\left(R^{2} \geq 0.9992\right)$. The RSDs of intra- and inter-day variations for 14analytes were not beyond $3.19 \%$ and $4.75 \%$, respectively. The LODs and LOQs ranged from 0.2 to $20 \mu \mathrm{g} / \mathrm{L}$ and from 0.5 to $30 \mu \mathrm{g} / \mathrm{L}$, respectively. The established method demonstrated acceptable accuracy with spike recovery of $96.75 \%-105.78 \%$ for all analytes; and the RSDs of the peak areas for 14analytes detected within $24 \mathrm{~h}$ were lower than $4.87 \%$. These results indicated that the developed UPLC-MS method was efficient, accurate and sensitive for simultaneous quantitative determination of the 14 constituents in WLJHT.

Table 2. Linear-regression data, LODs, LOQs and recovery of the 14 constituents determined by HPLC-MS/MS.

\begin{tabular}{|c|c|c|c|c|c|c|c|c|c|c|}
\hline \multirow[t]{2}{*}{ Analyte } & \multirow[t]{2}{*}{$\begin{array}{l}\text { EIC } \\
\text { Ions }\end{array}$} & \multirow[t]{2}{*}{ Regression Equation } & \multirow[t]{2}{*}{$\mathbf{R}^{2}$} & \multirow{2}{*}{$\begin{array}{l}\text { Linear } \\
\text { Range } \\
(\mu \mathrm{g} / \mathrm{L})\end{array}$} & \multirow[t]{2}{*}{$\begin{array}{l}\text { LOD } \\
(\mu \mathrm{g} / \mathrm{L})\end{array}$} & \multirow[t]{2}{*}{$\begin{array}{l}\mathrm{LOQ} \\
(\mu \mathrm{g} / \mathrm{L})\end{array}$} & \multicolumn{2}{|c|}{$\begin{array}{l}\text { Repeatability RSD } \\
(\%)(n=6)\end{array}$} & \multirow{2}{*}{$\begin{array}{c}\text { Stability } \\
\text { RSD (\%) } \\
(n=6)\end{array}$} & \multirow{2}{*}{$\begin{array}{l}\text { Standard Addition } \\
\text { Recovery }^{\text {a }}(\%) \text { Mean } \\
\pm \operatorname{SD}(n=6)\end{array}$} \\
\hline & & & & & & & Intra-Day & Inter-Day & & \\
\hline 4 & 153.1 & $\mathrm{y}=8599.2 \mathrm{x}+128.19$ & 0.9995 & $36 \sim 900$ & 6 & 30 & 1.56 & 1.85 & 2.35 & $96.75 \pm 1.57$ \\
\hline 5 & 137.1 & $y=37255 x+254.11$ & 0.9992 & $2.1 \sim 315$ & 1 & 5 & 2.14 & 2.45 & 2.68 & $99.57 \pm 2.77$ \\
\hline 9 & 353.1 & $y=15805 x+137.776$ & 0.9994 & 14.4 1440 & 5 & 10 & 2.63 & 3.85 & 1.46 & $101.17 \pm 2.53$ \\
\hline 10 & 179.1 & $y=20031 x+1383.5$ & 0.9995 & $30 \sim 3000$ & 20 & 30 & 2.98 & 3.42 & 4.87 & $103.51 \pm 1.01$ \\
\hline 11 & 609.1 & $y=13077 x+369.10$ & 0.9996 & $30 \sim 3000$ & 0.5 & 1 & 3.15 & 3.61 & 3.56 & $97.12 \pm 1.23$ \\
\hline 16 & 417.2 & $y=52008 x+417.68$ & 0.9995 & $2.4 \sim 480$ & 0.2 & 0.5 & 2.81 & 2.65 & 2.15 & $103.90 \pm 1.20$ \\
\hline 18 & 763.2 & $y=24665 x+78.310$ & 0.9998 & $4.08 \sim 408$ & 1 & 4 & 2.36 & 4.75 & 4.42 & $101.28 \pm 3.63$ \\
\hline 19 & 593.1 & $y=13603 x+559.91$ & 0.9993 & $48 \sim 2400$ & 0.5 & 1 & 2.25 & 3.47 & 2.49 & $99.24 \pm 3.36$ \\
\hline 20 & 623.2 & $y=16622 x+33.444$ & 0.9996 & $3.6 \sim 150$ & 0.5 & 1 & 1.93 & 2.38 & 3.52 & $97.96 \pm 2.20$ \\
\hline 22 & 447.1 & $y=27716 x+277.58$ & 0.9997 & $6 \sim 900$ & 0.2 & 1 & 3.19 & 4.28 & 1.94 & $98.53 \pm 3.21$ \\
\hline 23 & 515.1 & $y=7453.0 x-121.38$ & 0.9994 & $36 \sim 2700$ & 10 & 20 & 1.95 & 2.22 & 2.73 & $97.77 \pm 1.65$ \\
\hline 27 & 359.1 & $y=12867 x-23.768$ & 0.9996 & $12 \sim 1200$ & 5 & 10 & 2.48 & 3.42 & 3.72 & $97.57 \pm 1.19$ \\
\hline 30 & 1397.7 & $y=557.36 x+1.2437$ & 0.9994 & $10 \sim 120$ & 1 & 10 & 1.46 & 2.17 & 3.18 & $102.94 \pm 3.16$ \\
\hline 34 & 1073.6 & $\mathrm{y}=2184.1 \mathrm{x}+3.8435$ & 0.9992 & 3.6 120 & 1 & 2 & 2.85 & 3.14 & 2.65 & $105.78 \pm 4.12$ \\
\hline
\end{tabular}

\subsection{Quantitative Determination of the Major Constituents in the WLJHT}

The above HPLC-ELSD and HPLC-MS/MS methods were applied to quantify the contents of the 17 major constituents in 16 batches of WLJHT samples (Figure 3). All of the contents were calculated by the external standard method, and the mean values and SDs from the three parallel determinations of each sample are summarized in Table 3. In general, the total content of known chemical components reached $78.61 \%-90.06 \%$ of the dry weight of WLJHT samples. Among them, 14 representative non-sugar small molecules possessed $0.25 \% 0-0.29 \%$, and monosaccharide/sucrose accounted for $78.58 \%-90.03 \%$. These results exhibited a general feature of WLJHT's chemical profile: saccharides are the major components, and the non-sugar small molecules possess a very low content. 


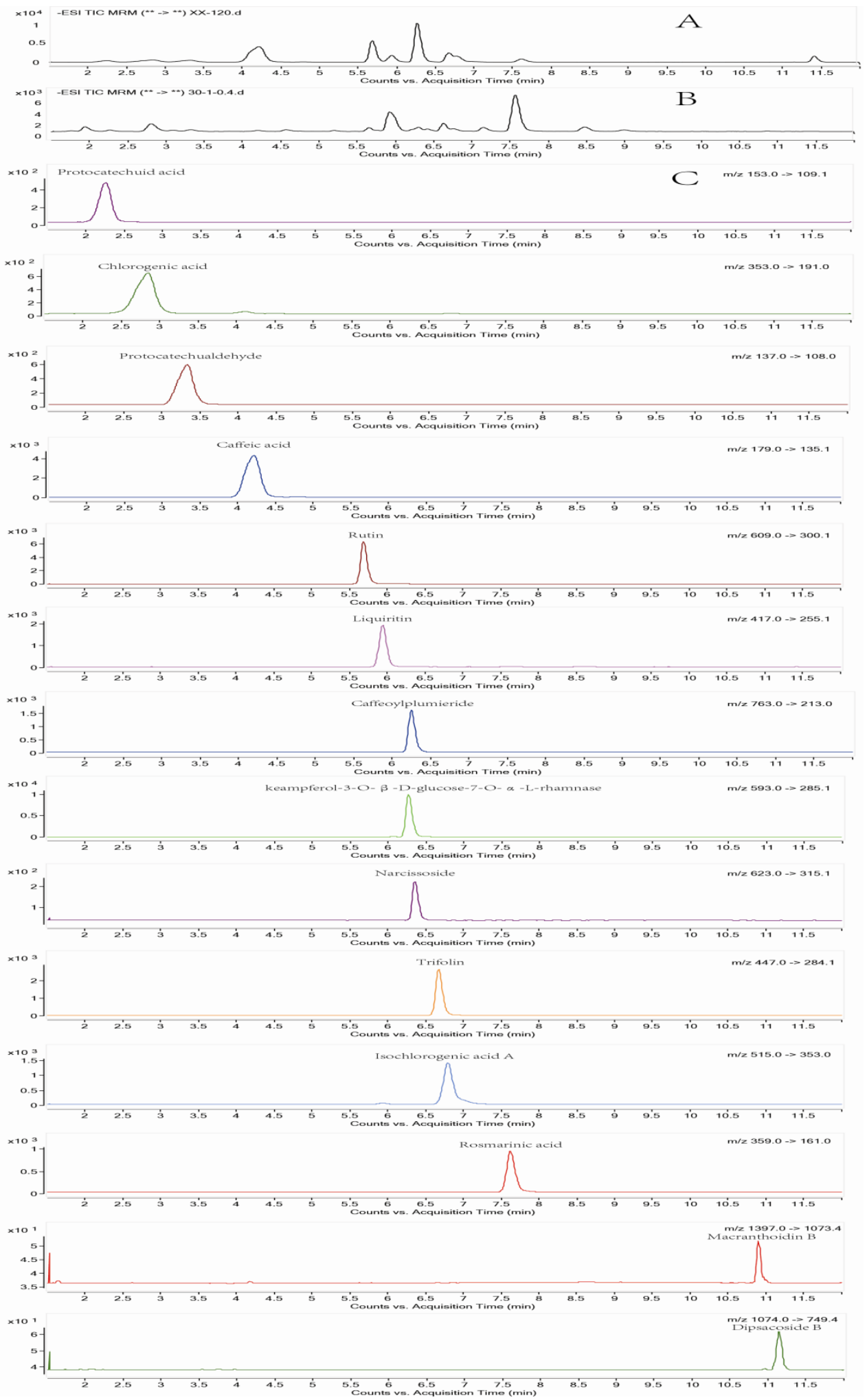

Figure 3. TIC chromatograms obtained by MRM for the negative-ion ESI triple quadrupole MS of 14 reference substance. (A) Extracted ion chromatograms of the fourteen reference substances; (B) Total ion chromatograms of the samples; (C) Extracted ion chromatograms obtained by MRM for negative-ion mode of the fourteen reference substances. 
Table 3. The contents of 17 analytes in 16 batches of WLJHT by HPLC-MS/MS and HPLC-ELSD ( $\mu \mathrm{g} / \mathrm{mL})$.

\begin{tabular}{|c|c|c|c|c|c|c|c|c|c|c|c|c|c|c|c|c|}
\hline Analyte & W141009 & W141011 & W141012 & W141013 & W141015 & W141021 & W141023 & W141024 & W141025 & W141026 & W141028 & W141030 & W141117 & W141118 & W141119 & W141120 \\
\hline 4 & 0.76 & 0.85 & 0.79 & 0.80 & 0.86 & 0.77 & 0.81 & 0.80 & 0.78 & 0.77 & 0.79 & 0.82 & 0.80 & 0.81 & 0.81 & 0.80 \\
\hline 5 & 0.62 & 0.64 & 0.61 & 0.60 & 0.65 & 0.63 & 0.56 & 0.63 & 0.57 & 0.57 & 0.58 & 0.57 & 0.57 & 0.57 & 0.56 & 0.54 \\
\hline 9 & 3.25 & 3.49 & 3.28 & 4.05 & 3.34 & 3.63 & 3.53 & 3.51 & 3.23 & 4.03 & 3.54 & 3.42 & 3.51 & 3.50 & 3.42 & 3.26 \\
\hline 10 & 0.54 & 0.53 & 0.55 & 0.56 & 0.56 & 0.62 & 0.58 & 0.56 & 0.51 & 0.55 & 0.52 & 0.54 & 0.46 & 0.48 & 0.49 & 0.43 \\
\hline 11 & 0.70 & 0.68 & 0.64 & 0.71 & 0.65 & 0.66 & 0.65 & 0.64 & 0.64 & 0.64 & 0.71 & 0.68 & 0.70 & 0.71 & 0.68 & 0.68 \\
\hline 16 & 3.46 & 3.72 & 3.55 & 3.29 & 3.61 & 3.34 & 3.14 & 3.24 & 3.13 & 3.23 & 3.54 & 3.23 & 3.26 & 3.14 & 3.06 & 2.96 \\
\hline 18 & 0.05 & 0.04 & 0.04 & 0.05 & 0.04 & 0.04 & 0.03 & 0.04 & 0.05 & 0.05 & 0.04 & 0.04 & 0.04 & 0.05 & 0.04 & 0.04 \\
\hline 19 & 0.66 & 0.64 & 0.58 & 0.66 & 0.58 & 0.63 & 0.64 & 0.62 & 0.61 & 0.57 & 0.63 & 0.64 & 0.73 & 0.68 & 0.62 & 0.63 \\
\hline 20 & 1.23 & 1.26 & 1.13 & 1.24 & 1.15 & 1.16 & 1.18 & 1.24 & 1.16 & 1.15 & 1.32 & 1.26 & 1.42 & 1.23 & 1.14 & 1.25 \\
\hline 22 & 1.45 & 1.43 & 1.36 & 1.54 & 1.37 & 1.56 & 1.53 & 1.58 & 1.56 & 1.57 & 1.46 & 1.51 & 1.57 & 1.69 & 1.56 & 1.41 \\
\hline 23 & 2.36 & 2.23 & 2.22 & 2.31 & 2.24 & 2.23 & 2.34 & 2.13 & 2.20 & 2.26 & 2.12 & 2.10 & 2.27 & 2.26 & 2.11 & 1.87 \\
\hline 27 & 9.65 & 9.13 & 9.11 & 10.28 & 9.22 & 9.54 & 9.58 & 9.23 & 10.12 & 10.07 & 9.85 & 9.35 & 9.22 & 10.15 & 9.49 & 8.78 \\
\hline 30 & 0.05 & 0.03 & 0.03 & 0.07 & 0.08 & 0.08 & 0.08 & 0.04 & 0.06 & 0.06 & 0.04 & 0.05 & 0.06 & 0.06 & 0.03 & 0.03 \\
\hline 34 & 0.03 & 0.01 & 0.04 & 0.02 & 0.02 & 0.02 & 0.02 & 0.01 & 0.01 & 0.02 & 0.02 & 0.01 & 0.02 & 0.01 & 0.03 & 0.02 \\
\hline Sub-total & 24.81 & 24.68 & 23.93 & 26.18 & 24.37 & 24.91 & 24.67 & 24.27 & 24.63 & 25.54 & 25.16 & 24.22 & 24.63 & 25.34 & 24.04 & 22.79 \\
\hline Fructose ${ }^{a}$ & 5.87 & 7.14 & 6.69 & 6.06 & 6.52 & 5.96 & 6.32 & 5.95 & 6.66 & 7.55 & 6.52 & 5.99 & 6.31 & 6.51 & 7.17 & 8.39 \\
\hline Glucose $^{a}$ & 7.44 & 8.20 & 7.50 & 7.67 & 7.56 & 8.29 & 8.32 & 8.11 & 7.65 & 7.44 & 8.44 & 7.20 & 8.23 & 7.96 & 7.25 & 7.69 \\
\hline Sucrose ${ }^{a}$ & 60.23 & 63.60 & 62.42 & 61.48 & 66.98 & 66.33 & 62.14 & 62.99 & 64.05 & 64.48 & 66.04 & 61.21 & 62.41 & 64.83 & 61.44 & 63.82 \\
\hline Dry weight a & 86.46 & 87.68 & 91.43 & 95.71 & 91.69 & 92.60 & 91.52 & 88.08 & 92.84 & 88.78 & 94.17 & 87.03 & 93.96 & 98.05 & 91.68 & 90.84 \\
\hline Content (\%) & 85.09 & 90.06 & 83.82 & 78.61 & 88.43 & 87.05 & 83.92 & 87.50 & 84.43 & 89.54 & 86.04 & 85.52 & 81.92 & 80.90 & 82.77 & 87.98 \\
\hline
\end{tabular}

a The unit of weight was $\mathrm{mg} / \mathrm{mL}$. 


\section{Materials and Methods}

\subsection{Materials and Reagents}

Acetonitrile and methanol of HPLC grade were purchased from Honeywell (Muskegon, MI, USA). Water was purified by a Milli-Q water-purification system (Milford, MA, USA). Formic acid and ethyl acetate was analytical grade and purchased from Guangzhou Chemical Reagent Factory (Guangzhou, China).

Fourteen reference substamces, protocatechuid acid (4), protocatechualdehyde (5), chlorogenic acid (9), caffeic acid (10), narcissoside (20), trifolin (22), isochlorogenic acid A (23) and rosmarinic acid (27) were purchased from Chengdu Must Bio-Technology Co., Ltd. (Chengdu, China). Rutin (11), liquiritin (16), keampferol-3-O- $\beta$-D-glucose-7-O- $\alpha$-L-rhamnase (19), macranthoidin B (30), and dipsacoside B (34) were purchased from Chengdu Push Bio-Technology Co., Ltd. (Sichuan, China). Caffeoylplumieride (18) were produced by our laboratory. Reference substances of D-(-)-fructose, D-(+)-glucose and sucrose were purchased from Sigma-Aldrich (St. Louis, MO, USA). Structures as shown in Figure 4 were elucidated based on their spectral analyses (IR, UV, MS and NMR), and their purities were found by HPLC analysis to be more than $98.0 \%$.

Sixteen batches of WLJHT were provided by Guangzhou Wanglaoji Pharmaceutical Co., Ltd. (Guangzhou, China). The batch number for each sample was W141009, W141011, W141012, W141013, W141015, W141021, W141023, W141024, W141025, W141026, W141028, W141030, W141117, W141118, W141119, and W141120. Sample W141117 was used for our method development studies.

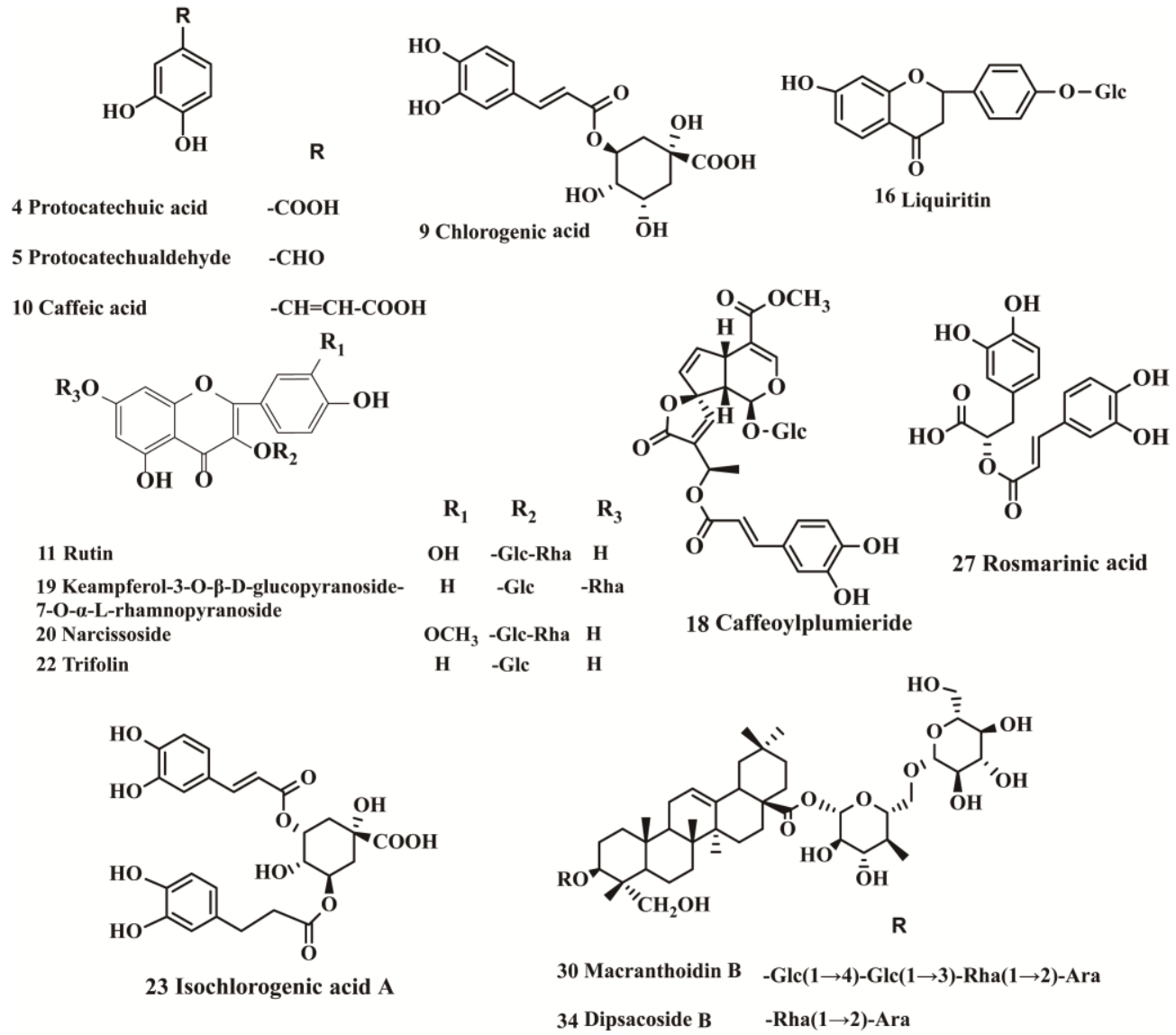

Figure 4. Chemical structures of the 14 reference substances. 


\subsection{Preparation of Reference Solutions}

Standard solutions of these 14 reference compounds were prepared in methanol-water $(3: 7, v / v)$ atthe known concentration $(\mathrm{mg} / \mathrm{mL})$ :protocatechuid acid (2.01), protocatechualdehyde (1.46), chlorogenic acid (2.40), caffeic acid (2.02), Rutin (1.06), liquiritin (1.03), Caffeoylplumieride(1.36), keampferol-3-O- $\beta$-D-glucose-7-O- $\alpha$-L-rhamnase (1.61), narcissoside (0.43), trifolin (0.42), isochlorogenic acid A (1.21), rosmarinic acid (1.98) macranthoidin B (0.21), and dipsacoside B (0.22). All standard solutions were stored at $4{ }^{\circ} \mathrm{C}$ until used, and finally filtered through a nylon membrane filter $(0.22 \mu \mathrm{m}$, Phenomenex, Los Angeles, CA, USA) before analysis.

\subsection{Preparation of Sample Solutions}

The samples were stored in a refrigerator at $4{ }^{\circ} \mathrm{C}$ until used, and they warmed to room temperature, and filtered through a nylon membrane filter $(0.22 \mu \mathrm{m}$, Phenomenex, USA) for qualitative analysis. For HPLC-MS/MS analysis, $1 \mathrm{~mL}$ of sample solution was transferred to 5-mL volumetric flask, brought up to volume with methanol-water $(3: 7, v / v)$ and filtered through a nylon membrane filter $(0.22 \mu \mathrm{m}$, Phenomenex, USA) prior to use.

\subsection{UPLC/Q-TOF-MS/MS Instrumentation and Methods}

UPLC/Q-TOF-MS analysis was performed using an AB Sciex 5600 Triple-TOFTM mass spectrometer (AB Sciex, Redwood, CA, USA) coupled to a Shimadzu UPLC LC-30AD system (Kyoto, Japan) which were controlled with an Analyst ${ }^{\circledR}$ TF 1.7 software (AB Sciex, Framingham, MA, USA). The chromatographic separations were accomplished on an ACQUITY UPLC BEH C18 column (Waters, Milford, MA, USA; $100 \times 2.1 \mathrm{~mm}, 1.7 \mu \mathrm{m}$ ) with the column temperature kept at $25^{\circ} \mathrm{C} .0 .1 \%$ formic acid (A) and acetonitrile (B) were served as the mobile phases at a flow rate of $0.35 \mathrm{~mL} / \mathrm{min}$ under the following gradient elution mode: 0-2 min, 5\% B; 2-3 min, 5\%-10\% B; 3-5 min, 10\% B; 5-11 min, $10 \%-30 \% \mathrm{~B} ; 11-20 \mathrm{~min}, 30 \%-60 \% \mathrm{~B} ; 20-25 \mathrm{~min}, 60 \%-95 \%$ B. The injection volume was $5 \mu \mathrm{L}$, the column temperature was at room temperature. The mass spectrometer coupled with an electrospray ionization (ESI) sources was run in negative/positive ion and high sensitivity mode to acquire the TOF-MS. Meanwhile, accurate mass measurements were acquired with an automated calibration delivery system. After optimization, the nebulizer gas, heater gas and curtain gas were set at 55, 55 and 35 psi, respectively, and nitrogen was used as the source gases. The source temperature, ion spray voltage and declustering potential were set at $500{ }^{\circ} \mathrm{C}, \pm 4500 \mathrm{~V}$ and $100 \mathrm{~V}$, respectively. For the IDA experiments, the collision energies were set at $-45 \mathrm{eV}$ and $-25 \mathrm{eV}$, and the collision energy spread was set at $15 \mathrm{eV}$. TOF-MS spectra were obtained from 100 to 1500 Da followed by information dependent acquisition (IDA) scanning from 50 to 2000 Da. For further review of the mass spectrometric data for qualitative analysis, PeakView 2.0 Software (AB Sciex, Framingham, MA, USA) was used.

\subsection{HPLC-ELSD Instrumentation and Methods}

A previously reported HPLC-HILIC-ELSD method was used to determine the monosaccharides and oligosaccharides in TCM samples [2]. For quantitation of D-(-)-fructose, D-(+)-glucose and sucrose, an Agilent 1260liquidchromatography system (Agilent Technologies, Palo Alto, CA, USA), and Alltech3300 evaporative light scattering detector (Grace Alltech, Deerfield, IL, USA) coupled with a Merck ZIC-HILIC (4.6 mm × $200 \mathrm{~mm}$, Merck, Tokyo, Germany) column at $30^{\circ} \mathrm{C}$ were used. Water (A) and acetonitrile (B) were served as the mobile phases at a flow rate of $1.0 \mathrm{~mL} / \mathrm{min}$ under the following gradient elution mode: 0-10 $\mathrm{min}, 85 \% \mathrm{~B} ; 10-20 \mathrm{~min}, 85 \%-70 \% \mathrm{~B} ; 20-40 \mathrm{~min}, 70 \%-55 \% \mathrm{~B}$. The injection volume was $10 \mu \mathrm{L}$, the drift tube temperature of ELSD was set at $60{ }^{\circ} \mathrm{C}$ and the nitrogen flow rate of ELSD was set at $1.8 \mathrm{~L} / \mathrm{min}$. The gain number was equal to 1 . 


\subsection{HPLC-MS/MS Instrumentation and Methods}

For quantitation of 14 major constituents in the WLJHT, the HPLC-MS/MS analysis was performed using an Agilent 1260 HPLC system, equipped with G1312B 1260 Bin Pump, G1367E 1260 Hip ALS, G1316A 1260 TCC, Agilent 6460 LC/QQQ, Chemstation online workstation, electrospray ion source (ESI) and Agilent Poroshell 120 EC- $\mathrm{C}_{18}$ column $(3.0 \mathrm{~mm} \times 50 \mathrm{~mm}, 2.7 \mu \mathrm{m}) .0 .1 \%$ formic acid (A) and acetonitrile (B) were served as the mobile phases at a flow rate of $0.25 \mathrm{~mL} / \mathrm{min}$ under the following gradient elution mode: $0-3 \mathrm{~min}, 13 \%-25 \% \mathrm{~B} ; 3-7 \mathrm{~min}, 25 \% \mathrm{~B} ; 7-8 \mathrm{~min}, 25 \%-40 \% \mathrm{~B}$; $8-12 \mathrm{~min}, 40 \%-54 \% \mathrm{~B}$. The injection volume was $5 \mu \mathrm{L}$, the column temperature was $25^{\circ} \mathrm{C}$. For MS condition, the capillary voltage was set at $3500 \mathrm{~V}$, nozzle voltage was $500 \mathrm{~V}$. Nebulizer air, drying-gas and sheath gas were all nitrogen, the drying-gas temperature was $300{ }^{\circ} \mathrm{C}$ and the flow rate was $5 \mathrm{~L} / \mathrm{min}$, the nebulizer pressure was $45 \mathrm{psi}$, sheath gas temperature was 300 and its flow rate was $11 \mathrm{~L} / \mathrm{min}$, the mass scanning range was set from $\mathrm{m} / z 100$ to 1500 . The optimum parameters of triple quadruple mass spectrometry are given in Table 2 .

\section{Conclusions}

In this study, UPLC/Q-TOF-MS/MS, HPLC-MS/MS and HPLC-ELSD methods were developed for the identification and determination of the major constituents in WLJHT. The UPLC coupled with MS quickly identified or tentatively characterized 34 compounds in WLJHT based on their determined exact molecular weights and fragmentation patterns. Accurate determinations of 17 major constituents in WLJHT were performed by HPLC-ELSD and HPLC-MS/MS methods, respectively. Compared to the reported method in the literature [20], the complex application of the above three methods showed good stability, reproducibility, comprehensiveness, and could be applied for the quality control of WLJHT.

Supplementary Materials: The following are available online.

Author Contributions: C.-Z.L. and C.-C.Z. conceived and designed the experiments; R.-J.Z. and B.-J.W. performed the experiments; C.-Z.L. and Y.-F.Y. analyzed the data; X.-D.H. and R.-B.Z. contributed reagents/materials; C.-Z.L. and R.-J.Z. wrote the paper; C.-C.Z. revised the paper.

Funding: This study was Financial supported by the National Natural Science Foundation of China (Nos. 81373928, 81460659, 81573566 and 81673872) and Department of Education Guangdong of Province (No. YQ2013043). It is also supported by Pearl River S\&T Nova Program of Guangzhou (No. 2012J2200001).

Conflicts of Interest: The authors declare no conflict of interest.

\section{References}

1. He, R.R.; Yao, X.S.; Kurihara, H. Studies on the Xiehuo effect and compositions of guangdong herbal tea. World Sci. Technol. 2009, 11, 834-839.

2. Bao, L.; Yao, X.S.; He, R.R.; Kurihara, H. Protective effects of Guangdong Liangcha grandes on restraint stress-induced liver damage in mice. J. Chin. Mater. Med. 2008, 33, 664-668.

3. Xie, X.G.; Chen, Y.; Bu, Y.Q.; Dai, C.C. A review of allelopathic researches on phenolic cids. Acta Ecol. Sin. 2014, 34, 6417-6428.

4. Li, S.B.; He, R.R.; Wang, M.; Xie, G.; Yao, X.S.; Kurihara, H. Effects of Wanglaoji Herbal Tea on Cytotoxic T Lymphocyte Activity in Spleen of Restraint Stress Mice. J. Chin. Med. 2010, 21, 223-226.

5. He, R.R.; Kurihara, H.; Bao, L.; Yao, X.S. The Effect of Wang Laoji Liangcha on Plasma Lipids Metabolism in restraint mice. Chin. J. Exp. Tradit. Med. Form. 2008, 14, 31-33.

6. He, R.R.; Kurihara, H.; Bao, L.; Li, M.M.; Yao, X.S. Effect of Wanglaoji Cool Tea on plasma glucometabolism and per-oxidative state in stress mice. Chin. Tradit. Pat. Med. 2008, 30, 1111-1114.

7. He, R.R.; Kurihara, H.; Bao, L.; Li, M.M.; Yao, X.S. Effect of Wang Laoji Liangcha on Immunologic Fanction and Oxidation in Restrained Mice. Chin. J. Exp. Tradit. Med. Form. 2008, 14, 38-42.

8. He, Y.Y.; Luo, Y.Y.; Lin, C.Z.; Lin, D.H.; Zhu, C.C.; He, H.L. Chemical constituents from Wanglaoji Herbal Tea. J. Chin. Med. 2018, 41, 889-893. 
9. Deng, J.W.; Fan, C.L.; Yang, Y.Y. Identification and determination of the major constituents in Deng's herbal tea granules by rapid resolution liquid chromatography coupled with mass spectrometry. J. Pharm. Biomed. Anal. 2011, 56, 928-936. [CrossRef] [PubMed]

10. Villiers, A.D.; Venter, P.; Pasch, H. Recent advances and trends in the liquid-chromatography-mass spectrometry analysis of flavonoids. J. Chromatogr. A 1430, 1430, 16-78. [CrossRef] [PubMed]

11. Bao, Y.W.; Li, C.; Shen, H.W.; Nan, F.J. Determination of saikosaponin derivatives in Radix bupleuri and in pharmaceuticals of the chinese multi herb remedy xiaochaihu-tang using liquid chromatographic tandem mass spectrometry. Anal. Chem. 2004, 76, 4208-4216. [CrossRef] [PubMed]

12. Wang, H.; Feng, F. Identification of components in Zhi-Zi-Da-Huang decoction by HPLC coupled with electrospray ionization tandem mass spectrometry, photodiode array and fluorescence detectors. J. Pharm. Biomed. Anal. 2009, 49, 1157-1165. [CrossRef] [PubMed]

13. Zou, H.Y.; Chen, Z.J.; Zheng, J.M. The application of GC-MS in Chinese herbal medinine. Mod. Bus. Trade Ind. 2010, 9, 362-364.

14. Chau, S.L.; Huang, Z.B.; Song, Y.G.; Yue, R.Q.; Ho, A.; Lin, C.Z.; Huang, W.H.; Han, Q.B. Comprehensive Quantitative Analysis of SQ Injection Using Multiple Chromatographic Technologies. Molecules 2016, 21, 1092. [CrossRef] [PubMed]

15. Tsuruoka, M.; Yamagata, K. Capillary electrophoresis-mass spectrometry-based metabolome analysis of serum and saliva from neurodegenerative dementia patients. Electrophoresis 2013, 34, 2865-2872. [CrossRef] [PubMed]

16. Ye, Z.; Dai, J.R.; Zhang, C.G.; Lu, Y.; Wu, L.L.; Gong, G.W.; Xu, H.; Tsim, W.K.; Wang, Z.T. Chemical Differentiation of Dendrobium officinale and Dendrobium devonianum by Using HPLC Fingerprints, HPLC-ESI-MS, and HPTLC Analyses. Evid.-Based Complement. Altern. Med. 2017, 13, 1-9. [CrossRef] [PubMed]

17. Carlier, J. The principal toxic glycosidic steroids in Cerberamanghas L. seeds: Identifiction of cerberin, neriifolin, tanghinin and deacetyltanghinin by UHPLC-HRMS/MS, quantification by UHPLC-PDA-MS. J. Chromatogr. B 2014, 926, 1-8. [CrossRef] [PubMed]

18. Zhou, Y.; Wang, M.K.; Liao, X.; Zhu, X.M.; Peng, S.L.; Ding, L.S. Rapid identification of compounds in Glycyrrhiza Uralensis by liquid chromatography/tandem mass spectrometry. Chin. J. Anal. Chem. 2004, 32, 174-178.

19. Zhang, Q.L.; Li, J.; Wang, C.; Sun, W.; Zhang, Z.T.; Cheng, W.M. A gradient HPLC method for the quality control of chlorogenic acid, linarin and luteolin in Flos Chrysanthemi Indici suppository. J. Pharm. Biomed. Anal. 2007, 43, 753-757. [CrossRef] [PubMed]

20. Zhu, P.C.; Cen, W.J.; Fan, X.S. Simultaneous determination of three effective components in Wanglaoji cool tea by ultra performance liquid chromatography and tandem mass spectroscopy. J. Hubei Univ. 2014, $36,123-126$.

Sample Availability: Samples of the compounds are not available from the authors.

(C) 2018 by the authors. Licensee MDPI, Basel, Switzerland. This article is an open access article distributed under the terms and conditions of the Creative Commons Attribution (CC BY) license (http:/ / creativecommons.org/licenses/by/4.0/). 